although $2.5 \mathrm{mgm}$. per cent can inhibit carbonic anhydrase by nearly 50 per cent, it can also stabilize the enzyme. Now even a slight degree of stabilization means that the enzyme will act catalytically in the boat for a longer period of shaking. Hence any estimation of activity which is based on the total amount of gas liberated over a given period may be very much in error. It is even possible for the stabilization to be so pronounced that such a method of estimation would indicate some degree of 'activation', whereas there was actually an inhibition. This kind of error is readily avoided by using the unimolecular velocity constant in the manner described.

This work has been carried out during the tenure of an 1851 Exhibition Research Scholarship. My thanks are due to Mr. Levenbook for assistance with the electrometric experiments.

Molteno Institute,

A. M. ClaRK Cambridge.

' Wilbur and Anderson, J. Biol. Chem., 178, 147 (1948).

'Klese and Hastings, J. Biol. Chem., 132, 281 (1940).

'Meldrum and Roughton, J. Physiol., 80, 113 (1934).

"Mitchell, Pozzani and F'essenden, J. Biol. Chem., 160, 283 (1945).

- Scott and Mendive, J. Biol. Chem., 189, 661 (1941).

\section{Effect of Urethane Combined with X-Rays on Chick Fibroblasts}

THE similarity of action between urethane (ethyl carbamate) and X-rays suggests that a common mechanism of action may be present. The similarity has been seen in several ways. For example, both agents have been found to inhibit mitosis, the arrest primarily affecting the prophase stage of division. The similarity is also seen in the clinical treatment of human leukrmia, where the effects of X-rays and of urethane seem indistinguishable.

We have also found that chick fibroblasts treated with urethane show chromosome fragmentation and 'stickiness' resembling closely the appearances produced by X-rays; see accompanying photographs of cells fixed after an $80-\mathrm{min}$. exposure to $0 \cdot 2$ per cent

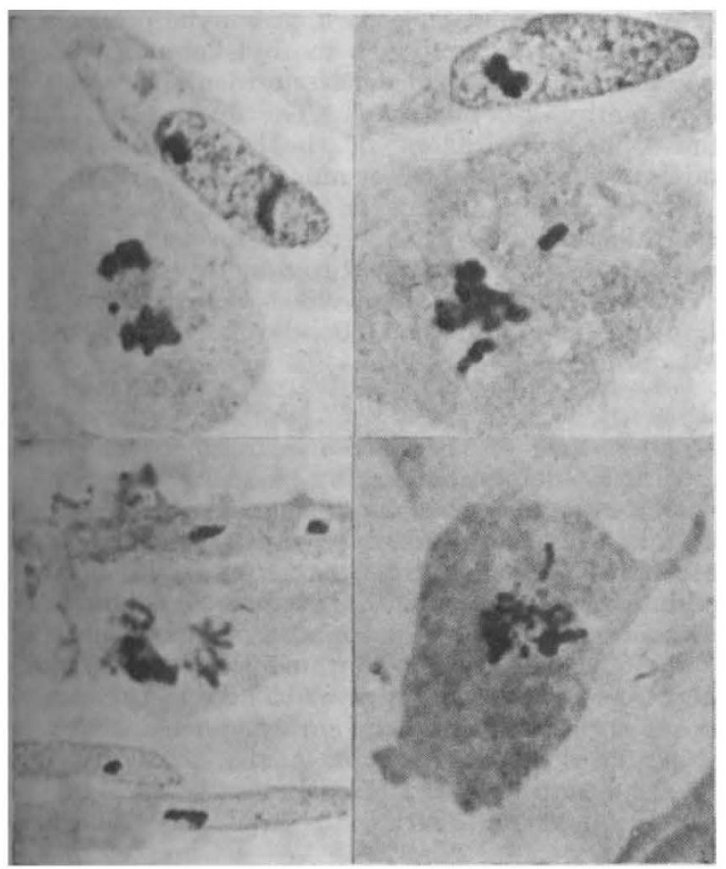

urethane. Chromosome abnormalities have previously been reported for plant material by Oehlkers'.

It might be expected that, if the two agents acted through a similar mechanism, a combination of urethane and X-rays would have a greater effect than that of either agent used alone. This has been tested and is not the case. Using the inhibition of mitosis in fibroblasts as a criterion, it has been found that the effect of a dose of 30 r. X-rays is diminished if urethane is added to the culture medium in a concentration of 0.1 per cent, which would itself reduce mitosis significantly. The reduction found with the combined treatment is less than that produced by X-rays alone, but is usually greater than that produced by urethane in the concentration used. The reduction occurs whether urethane is added before or up to a period of at least $40 \mathrm{~min}$. after the irradiation. This diminished effect is also reflected in a lowered number of chromosome aberrations found as a result of the combined treatment.

In view of the possibility that the findings might depend on a shift of the mitotic cycle giving the appearance of diminished effect, experiments have been made using the criterion of delayed lethal action on fibroblasts. A dose of 6,000 r. was given in fractions. Just before each irradiation session, a solution containing 0.2 per cent urethane in Tyrode was added to the medium. Cultures so treated showed a sig. nificantly higher survival-rate than those treated with Tyrode solution and X-rays.

Three conclusions seem to be justified : (I) X-ray action is incomplete at the time of irradiation, and is capable of being reversed at least in part up to a considerable time after it has been administered. (2) While urethane by itself produces effects characteristic of $\mathrm{X}$-rays, it also can partially reverse an $\mathrm{X}$-ray effect when the two agents are combined. This finding does not necessarily prove that the action of the two agents is exerted on a different chain of events in the cell, but it suggests this possibility. The experiments could, however, be interpreted on a hypothesis that the mechanism was the same but that the diminution of X-ray effect following urethane was due to a separate property of urethane, possibly the depression of respiration. (3) The experimental evidence provides no basis for expecting that an augmented effect would be obtained in the treatment of leukæmia by the simultaneous use of X-rays and urethane.

The detailed experiments will be published elsewhere.

\section{Edith Paterson} MARY V. THOMPSON

Department of Radiobiology,

Christie Hospital and Holt Radium Institute, Manchester 20 . Oct. 6.

${ }^{1}$ Oehlkers, F., Z. indukt. Abstam. und Vererb., 81, 313 (1943).

\section{A New Method for Determining the Weight of Cellular Structures}

SUBSTITUTION of a continuous spectrum for monochromatic X-rays in the methods now being used for cytochemical elementary analysis ${ }^{1,2}$ makes it possible to weigh single cell structures when a sufficiently broad band of a continuous X-ray spectrum is used. The effects of the absorption jumps of the elements in the tissue can be neglected, and the total absorption is proportional to the total mass of tissue. The wave-lengths of the chosen band must be selected properly. 